\title{
ON GENERALISING THE NOTION OF FIBRE SPACES TO INCLUDE THE FIBRE BUNDLES ${ }^{1}$
}

\section{SZE-TSEN HU}

1. Introduction. There are two different notions of fibering that have been investigated in the realm of topology. The one is the notion of a fibre bundle, defined by Whitney $[11]^{2}$ and improved by Steenrod [8]; the other is that of a fibre space, introduced by HurewiczSteenrod [6] and generalized by Fox [3]. These two notions do not coincide, as remarked by Steenrod, $[8$, p. 302]; there are fibre spaces that are not fibre bundles, while it is still unsolved whether every fibre bundle is a fibre space.

According to Fox [3, p. 555], the object of introducing the definition of fibre spaces is to state a minimum set of readily verifiable conditions under which the covering homotopy theorem holds. It seems to the author that this set of conditions; given by Hurewicz-Steenrod [6] and Fox [3], is not minimum in the sense that it does not naturally apply to the fibre bundles for which the covering homotopy theorem was proved by Steenrod [8, p. 303] in a somewhat weaker form. This weak theorem of covering homotopy of Steenrod works satisfactorily in nearly all the applications where the base space is normal Hausdorff.

In order that the weak theorem of covering homotopy hold, the existence of a unified slicing function in the definition of HurewiczSteenrod [6] and in that of Fox [3] is unnecessarily strong. The object of the present paper is to give a generalization of the notion of a fibre space by localizing the slicing function. It will be proved that the generalized class of fibre spaces will include all fibre bundles and that the weak theorem of covering homotopy will still hold.

As mentioned above, there are two forms of the covering homotopy theorem. In the "weak" form, the set being deformed is compact and the homotopy is unrestricted. In the "strong" form, the set is unrestricted but the homotopy is required to be uniform. Since any homotopy of a compact space is uniform, the "strong" theorem implies the "weak" theorem. If the set is completely regular, any uniform homotopy into a compact Hausdorff space can be extended to

Presented to the Society, February 25, 1950; received by the editors September $14,1949$.

1 This work was done under Contract N7-onr-434, Task Order No. III, Navy Department, Office of Naval Research.

2 Numbers in brackets refer to the bibliography at the end of the paper. 
a homotopy of its compaction. Therefore, in this case, the "strong" theorem follows from the "weak" theorem. Thus the two theorems are entirely equivalent for compact Hausdorff fibre spaces over Hausdorff base spaces when the set being deformed is completely regular. These relations are studied in $\$ 8 .^{8}$

2. Fiberings. By a fibering $F$ of a topological space $X$, we understand the collection

$$
F=\left\{X, B, \Omega, \pi, \phi_{U}\right\}
$$

of the following five entities:

(1) The topological space $X$, called the total space;

(2) A topological space $B$, called the base space;

(3) A system $\Omega=\{U\}$ of open sets $U$ of $B$ which covers $B$, called the slicing neighborhoods;

(4) A continuous map $\pi: X \rightarrow B$ of $X$ onto $B$, called the projection;

(5) A system of continuous maps $\left\{\phi_{U}\right\}$ indexed by $U \in \Omega$, called the slicing functions, where each $\phi_{U}$ is defined on the subset $U$ $\times \pi^{-1}(U)$ of the product space $B \times X$ with images in the total space $X$ in such a way that the following two conditions be satisfied:

$$
\begin{array}{rlrl}
\pi \phi_{U}(b, x) & =b, & (b \in U, & \left.x \in \pi^{-1}(U)\right) ; \\
\phi_{U}(\pi(x), x) & =x, & \left(x \in \pi^{-1}(U)\right) .
\end{array}
$$

If a fibering $F=\left\{X, B, \Omega, \pi, \phi_{U}\right\}$ of a given topological space $X$ exists, $X$ will be called a (generalized) fibre space over $B$ relative to $\pi$ and $\pi$ is said to be a fibre mapping. The following statement is an immediate consequence of the above definition and indicates clearly its local character:

(2.3) A topological space $X$ is a (generalized) fibre space over $B$ relative to a continuous onto map $\pi: X \rightarrow B$, if and only if, for each point $b \in B$, there exist an open set $U$ of $B$ containing $b$ and a continuous map $\phi_{U}: U \times \pi^{-1}(U) \rightarrow X$ such that the conditions (2.1) and (2.2) are satisfied.

By means of the conditions (2.1) and (2.2), the following statement is easy to prove:

(2.4) Every fibre mapping is open, that is, it maps open sets onto such.

3. Fiberings with a unified slicing function. A fibering $F$ $=\left\{X, B, \Omega, \pi, \phi_{U}\right\}$ is said to have a unified slicing function, if, for

${ }^{3}$ The author acknowledges his gratitude to the referee for his suggestions of these relations. In particular, the statements (8.2) and (8.3) were pointed out to the author by the referee. 
any two slicing neighborhoods $U$ and $V$, we always have $\phi_{U}=\phi_{V}$ on the intersection of $U \times \pi^{-1}(U)$ and $V \times \pi^{-1}(V)$. For such a fibering $F$, we may define a unified slicing function $\phi$ on the union $W$ of the subsets $U \times \pi^{-1}(U)$ for all slicing neighborhoods $U \in \Omega$ by taking $\phi=\phi_{U}$ on each $U \times \pi^{-1}(U)$ and denote the fibering by $F=\{X, B, \Omega, \pi, \phi\}$.

The following theorem is not difficult to prove:

(3.1) A topological space $X$ is a fibre space over $B$ relative to $\pi: X \rightarrow B$ in the sense of Fox [3, p. 556] if and only if there exists a fibering $F=\{X, B, \Omega, \pi, \phi\}$ with a unified slicing function.

We propose to call a fibre space in the sense of Fox (or in the sense of Hurewicz-Steenrod) a fibre space with a unified slicing function and to use the term "fibre space" in the generalized sense.

4. Homotopy type of the fibres. Let $X$ be a fibre space over $B$ relative to the projection $\pi: X \rightarrow B$. For every point $b \in B$, the subset $\pi^{-1}(b)$ of $X$ is called the fibre over $b$. We shall prove the following theorem.

(4.1) If two points $a, b \in B$ can be connected by $a$ path in $B$, then $\pi^{-1}(a)$ and $\pi^{-1}(b)$ have the same homotopy type.

In case the fibre space $X$ has a unified slicing function, this theorem was proved by Hurewicz-Steenrod [6, p. 62]. In their proof, however, an application of the strong theorem of covering homotopy [6, Theorem 1] was made. Since it is still unknown whether the strong theorem of covering homotopy holds for general fibre spaces, we have to give a direct proof as follows.

Proof. Let $F=\left\{X, B, \Omega, \pi, \phi_{U}\right\}$ be a fibering of $X$. According to our hypothesis, there is a continuous path $\sigma: I \rightarrow B$, where $I$ denotes the closed unit segment $\langle 0,1\rangle$ of real numbers, such that $\sigma(0)=a$ and $\sigma(1)=b$. It follows from the compactness of $I$ that there are a finite number of real numbers.

$$
0=t_{0}<t_{1}<\cdots<t_{n}=1
$$

such that, for each closed subsegment $I_{i}=\left\langle t_{i-1}, t_{i}\right\rangle(i=1,2, \cdots, n)$, there is a slicing neighborhood $U_{i}$ such that $\sigma\left(I_{i}\right) \subset U_{i}$. Hence, in order to prove (4.1), we can assume without loss of generality that $\sigma$ maps $I$ into some slicing neighborhood $U \in \Omega$.

Now we assume that $\sigma(I) \subset U$. Define two maps

$$
f: \quad \pi^{-1}(a) \rightarrow \pi^{-1}(b), \quad g: \quad \pi^{-1}(b) \rightarrow \pi^{-1}(a)
$$

by means of the formula

$$
\begin{aligned}
& f(x)=\phi_{U}(b, x), \\
& \left(x \in \pi^{-1}(a)\right) \text {, } \\
& g(x)=\phi_{U}(a, x), \\
& \left(x \in \pi^{-1}(b)\right) \text {. }
\end{aligned}
$$


It remains to prove that both $g f$ and $f g$ are homotopic with the identities. Let $\iota$ denote the identity map on $\pi^{-1}(a)$. To prove that $g f \simeq l$, let us consider the homotopy

$$
h_{t}: \quad \pi^{-1}(a) \rightarrow \pi^{-1}(a), \quad(0 \leqq t \leqq 1),
$$

defined by taking

$$
h_{t}(x)=\phi_{U}\left(a, \phi_{U}(\sigma(1-t), x)\right), \quad\left(x \in \pi^{-1}(a), 0 \leqq t \leqq 1\right) .
$$

Then we have $h_{0}=g f$ and $h_{1}=\iota$. Hence $g f \simeq \iota$. Similarly, one may prove that $f g$ is homotopic with the identity map on $\pi^{-1}(b)$. This completes the proof.

5. Fibre bundles. To prove that the total space of every fibre bundle be a fibre space, we shall recall, in the present section, the definition of a fibre bundle (see [11], [8], [2]).

By a fibre bundle $\Phi$, we understand the collection

$$
\Phi=\left\{X, B, \Omega, \pi, D, G, \psi_{U}\right\}
$$

of the following seven entities:

(1) A topological space $X$, called the total space;

(2) A topological space $B$, called the base space;

(3) A system $\Omega=\{U\}$ of open sets $U$ of $B$ which covers $B$, called the coordinate neighborhoods;

(4) A continuous map $\pi: X \rightarrow B$ of $X$ onto $B$, called the projection;

(5) A topological space $D$, called the director space;

(6) A topological group $G$ of homeomorphisms of $D$, called the reference group, topologized in such a way that the transformation $G \times D \rightarrow D$ defined by the operations of $G$ on $D$ is a continuous map;

(7) A system of homeomorphisms $\left\{\psi_{U}\right\}$ indexed by $U \in \Omega$, called the coordinate functions, where each function $\psi_{U}$ is a homeomorphism of the product space $U \times D$ onto $\pi^{-1}(U) \subset X$ satisfying the condition:

$$
\pi \psi_{U}(b, d)=b, \quad(b \in B, d \in D) .
$$

Further, these seven entities are supposed to satisfy the following

(5.2) Paste Condition. For a given coordinate neighborhood $U \in \Omega$ and a given point $b \in U$, denote by $\psi_{U, b}$ the homeomorphism of $D$ onto $\pi^{-1}(b)$ defined by $\psi_{U, b}(d)=\psi_{U}(b, d)$ for each $d \in D$. If $b$ belongs to two coordinate neighborhoods $U$ and $V$, then $\psi_{V, b}^{-1} \psi_{U, b}$ belongs to $G$ and depends continuously on $b \in U \cap V$.

6. The fibering associated with a fibre bundle. Let

$$
\Phi=\left\{X, B, \Omega, \pi, D, G, \psi_{U}\right\}
$$


be a given fibre bundle. We are going to construct a fibering

$$
F=\left\{X, B, \Omega, \pi, \phi_{U}\right\},
$$

which will be called the fibering associated with $\Phi$.

In order to construct $F$, it remains only to define the slicing functions $\phi_{U}$. Let $\xi_{U}: U \times D \rightarrow D$ be the projection defined by $\xi_{U}(b, d)=d$ for each $b \in U$ and each $d \in D$. Then $\phi_{U}$ is defined by

$$
\phi_{U}(b, x)=\psi_{U}\left(b, \xi_{U} \psi_{U}^{-1}(x)\right), \quad\left(b \in U, x \in \pi^{-1}(U)\right) .
$$

The conditions (2.1) and (2.2) are verified as follows:

$$
\begin{aligned}
\pi \phi_{U}(b, x) & =\pi \psi_{U}\left(b, \xi_{U} \psi_{U}^{-1}(x)\right)=b, & \left(b \in U, x \in \pi^{-1}(U)\right) ; \\
\phi_{U}(\pi(x), b) & =\psi_{U, \pi(x)} \psi_{U, \pi(x)}^{-1}(x)=x, & \left(x \in \pi^{-1}(U)\right) .
\end{aligned}
$$

The following theorem is an immediate consequence of our associated fibering.

(6.1) The total space $X$ of any fibre bundle $\Phi=\{X, B, \Omega, \pi, D$, $\left.G, \psi_{U}\right\}$ is a fibre space over $B$ relative to $\pi$.

7. The weak theorem of covering homotopy. With a few almost obvious modifications of the arguments of Steenrod, [8, p. 303], one can prove the weak theorem of covering homotopy for our generalized fibre spaces given as follows:

(7.1) If $X$ is a fibre space over a normal Hausdorff base space $B$ relative to a projection $\pi: X \rightarrow B, f: S \rightarrow X$ a continuous map of a compact topological space $S$ into $X$, and $h_{t}: S \rightarrow B(0 \leqq t \leqq 1)$ a homotopy with $h_{0}=\pi f$, then there exists a covering homotopy $f_{t}: S \rightarrow X(0 \leqq t \leqq 1)$ such that $f_{0}=f$ and $\pi f_{t}=h_{t}$ for each $0 \leqq t \leqq 1$. Further, we may assume that, if $h_{t}$ leaves $s_{0} \in S$ fixed so does $f_{t}$.

The following well known homotopy relations in fibre spaces and in fibre bundles are consequences of (7.1):

(7.2) If $X$ is a fibre space over a normal Hausdorff base space $B$ with pathwise connected fibres, then a necessary and sufficient condition for $X$ to be pathwise connected is the pathwise connectedness of the base space $B$.

(7.3) If $X$ is a pathwise connected fibre space over a normal Hausdorff base space $B$ with pathwise connected fibres and $X_{0}=\pi^{-1}\left(b_{0}\right)$ the fibre over $b_{0} \in B$, then the projection $\pi:\left(X, X_{0}\right) \rightarrow\left(B, b_{0}\right)$ induces (onto) isomorphisms of the homotopy groups

$$
\pi^{*}: \pi_{n}\left(X, X_{0}\right) \rightarrow \pi_{n}\left(B, b_{0}\right), \quad(n \geqq 2) .
$$

The following interesting statement is a consequence of the weak 
theorem of covering homotopy and a converse theorem of Fox $[4, \mathrm{p}$. 734]:

(7.4) Every compact fibre space $X$ over an absolute neighborhood retract $B$ has a unified slicing function.

8. Uniform homotopy and the strong theorem of covering homotopy. In the present section, the relations between the two forms of the covering homotopy theorem will be clarified. ${ }^{3}$

Let $B$ be a topological space and $\Omega=\{U\}$ be an open covering of $B$. A homotopy $h_{t}: S \rightarrow B(0 \leqq t \leqq 1)$ of a topological space $S$ into $B$ is said to be uniform with respect to the covering $\Omega$ if there exists a positive real number $\delta$ such that, for each $s_{0} \in S$ and $t_{0} \in I$, there corresponds an open set $U_{0} \in \Omega$ such that $h_{t}\left(s_{0}\right) \in U_{0}$ for each $t \in I$ satisfying the condition $\left|t-t_{0}\right| \leqq \delta$. $h_{t}$ is said to be uniform if it is uniform with respect to every open covering of $B$.

The strong theorem of covering homotopy for fibre spaces with a unified slicing function $[6$, p. $62 ; 3$, p. 557] can be stated as follows:

(8.1) Let $F=\{X, B, \Omega, \pi, \phi\}$ be a fibering of $X$ with a unified slicing function $\phi$. If $f: S \rightarrow X$ is a continuous map of a topological space $S$ into $X$, and $h_{t}: S \rightarrow B(0 \leqq t \leqq 1)$ a homotopy which is uniform with respect to $\Omega$ and satisfies the relation $h_{0}=\pi f$, then there exists a covering homotopy $f_{t}: S \rightarrow X(0 \leqq t \leqq 1)$ such that $f_{0}=f$ and $\pi f_{t}=h_{t}$ for each $0 \leqq t \leqq 1$. Further, we may assume that if $h_{t}$ leaves $s_{0} \in S$ fixed, so does $f_{t}$.

To discuss the strong theorem of covering homotopy for general fibre spaces, assume that $B$ be a compact Hausdorff space and $S$ a completely regular space. Let us denote the compaction of $S$ by the usual notation $\beta S$ (see [9], [1], [10], [7], and [5, p. 53]). We shall prove the following lemma.

(8.2) Every uniform homotopy $h_{t}: S \rightarrow B(0 \leqq t \leqq 1)$ can be extended to homotopy $h_{t}^{*}: \beta S \rightarrow B(0 \leqq t \leqq 1)$.

Proof. Imbed $B$ as a subset of the Tychonoff cube $Q$. The compactness of $B$ implies that $B$ is closed in $Q$. Since $S$ is dense in $\beta S$ and every bounded continuous real function defined on $S$ can be continuously extended throughout $\beta S$, it follows easily that, for every fixed $t$, the map $h_{t}: S \rightarrow B$ has a unique extension $h_{t}^{*}: \beta S \rightarrow B$. Since the given homotopy $h_{t}: S \rightarrow B(0 \leqq t \leqq 1)$ is uniform, it is not difficult to prove that the family $h_{i}^{*}: \beta S \rightarrow B(0 \leqq t \leqq 1)$ of maps form a homotopy. This completes the proof.

Now we are going to prove the following strong theorem of covering homotopy for compact Hausdorff fibre spaces.

(8.3) Let $F=\left\{X, B, \Omega, \pi, \phi_{U}\right\}$ be a fibering of a compact Hausdorff space $X$ over a Hausdorff base space $B$. If $f: S \rightarrow X$ be a continuous map 
of a completely regular space $S$ into $X$, and $h_{t}: S \rightarrow B(0 \leqq t \leqq 1)$ a uniform homotopy such that $h_{0}=\pi f$, then there exists a covering homotopy $f_{t}: S \rightarrow X(0 \leqq t \leqq 1)$ such that $f_{0}=f$ and $\pi f_{t}=h_{t}$ for each $0 \leqq t \leqq 1$. Further, we may assume that if $h_{t}$ leaves $s_{0} \in S$ fixed so does $f_{t}$.

Proof. Let $\beta S$ denote the compaction of $S$. Since $X$ is a compact Hausdorff space, the map $f: S \rightarrow X$ has a unique extension $f^{*}: \beta S \rightarrow X$. Since $B=\pi(X)$, the compactness of $X$ implies that of $B$. According to (8.2), the uniform homotopy $h_{t}: S \rightarrow B(0 \leqq t \leqq 1)$ has a unique extension $h_{t}^{*}: \beta S \rightarrow B(0 \leqq t \leqq 1)$. It is easy to see that $h_{0}^{*}=\pi f$. As a compact Hausdorff space, $B$ is normal. It follows from the weak theorem (7.1) that there exists a covering homotopy $f_{i}^{*}: \beta S \rightarrow X(0 \leqq t \leqq 1)$ such that $f_{0}^{*}=f^{*}$ and $\pi f_{i}^{*}=h_{t}^{*}$ for each $0 \leqq t \leqq 1$. Further, we may assume that if $h_{t}$ leaves $s_{0} \in S$ fixed so does $f_{t}^{*}$. The required covering homotopy $f_{t}: S \rightarrow X(0 \leqq t \leqq 1)$ is given by $f_{t}=f_{t}^{*} \mid S$ for each $0 \leqq t \leqq 1$. This completes the proof.

\section{BiBLIOGRAPHY}

1. E. Cech, On bicompact spaces, Ann. of Math. vol. 38 (1937) pp. 823-844.

2. S. S. Chern and Y. F. Sun, The imbedding theorem for fibre bundles, Trans. Amer. Math. Soc. vol. 67 (1949) pp. 286-303.

3. R. H. Fox, On fibre spaces. I, Bull. Amer. Math. Soc. vol. 49 (1943) pp. 555557.

4. - On fibre spaces. II, Bull. Amer. Math. Soc. vol. 49 (1943) pp. 733-735.

5. E. Hewitt, Rings of real-valued continuous functions. I, Trans. Amer. Math. Soc. vol. 64 (1948) pp. 45-99.

6. W. Hurewicz and N. E. Steenrod, Homotopy relations in fibre spaces, Proc. Nat. Acad. Sci. U.S.A. vol. 27 (1941) pp. 60-64.

7. I. Gelfand and G. E. Shilov, Ueber verschiedene Methoden der Einführung der Topologie in die Menge der maximalen Idealen eines normierten Ringes, Rec. Mat. (Mat. Sbornik) N.S. vol. 9 (1941) pp. 25-38.

8. N. E. Steenrod, The classification of sphere bundles, Ann. of Math. vol. 45 (1944) pp. 294-311.

9. M. H. Stone, Applications of the theory of Boolean rings to general topology, Trans. Amer. Math. Soc. vol. 41 (1937) pp. 375-481.

10. H. Wallman, Lattices and topological spaces, Ann. of Math. vol. 39 (1938) pp. 112-126.

11. H. Whitney On the theory of sphere-bundles, Proc. Nat. Acad. Sci. U.S.A. vol. 26 (1940) pp. 148-153.

Tulane University of Louisiana 